\title{
REMARKS ON POLYELECTROLYTE CONFORMATION
}

\author{
P. G. DE GENNES, P. PINCUS $\left({ }^{*}\right)$ and R. M. VELASCO (**) \\ Physique de la Matière condensée, Collège de France \\ 75231 Paris Cedex 05, France
}

and

\section{F. BROCHARD}

Bâtiment 510, Université Paris-Sud, 91405 Orsay, France

(Reçu le 3 mai 1976, révisé le 9 juillet 1976, accepté le 12 juillet 1976)

\begin{abstract}
Résumé. - Nous discutons des conformations de polymères linéaires chargés en faisant les hypothèses suivantes : $a$ ) la chaîne sans charge est flexible, $b$ ) la force électrostatique domine les
\end{abstract} interactions monomère-monomère, $c$ ) il n'y a pas de sels.

1) Pour le cas dilué (chaînes non enchevêtrées) en corrigeant le calcul self-consistant fait récemment par Richmond [1a], on trouve une taille des polyions égale à $R=N d$, qui est une fonction linéaire de l'indice de polymérisation $N$. Ce résultat est en accord avec les précédents travaux de Hermans et Overbeek [ $1 b]$, Kuhn, Kunzle et Katchalsky [1c].

2) Il existe un domaine pour des concentrations très petites $c\left(c^{* *}<c<c^{*}\right)$ où les interactions électrostatiques entre les polyions sont supérieures aux énergies thermiques, il semble donc possible que les polyions puissent former un réseau périodique à trois dimensions. Néanmoins, il semble difficile de mettre en évidence un réseau si dilué.

3) Jusqu'ici toutes les expériences avec les polyélectrolytes sans sels ont été pratiquement faites à des concentrations $c>c^{*}$, pour lesquelles les différentes chaînes sont enchevêtrées. Pour discuter ce régime on s'intéresse uniquement au cas où la charge par unité de longueur est près du (ou audessus du) seuil de condensation, donc il existe une seule longueur $\xi(c)$ caractérisant les corrélations ; à trois dimensions $\xi$ a le même comportement que le rayon de Debye pour les contre-ions. On a considéré quelques conformations possibles : $a$ ) un réseau hexagonal de bâtonnets; $b$ ) un réseau cubique de bâtonnets; $c$ ) une phase isotrope de chaînes partiellement flexibles. Les différentes structures formées de bâtonnets semblent avoir la même énergie électrostatique. Ce fait suggère que la phase isotrope peut être la plus favorable. On analyse cette dernière phase en utilisant les mêmes méthodes qui se sont révélées efficaces pour les solutions des polymères neutres. Dans le modèle isotrope chaque chaîne a le comportement d'une succession des petites pelotes (blobs) de taille $\xi$. Les effets électrostatiques sont importants à l'intérieur d'un blob et analogues au cas (1). Mais ces interactions sont écrantées entre les blobs; chaque chaîne a un comportement idéal à grande échelle et son rayon est $R(c) \sim c^{-1 / 4} N^{1 / 2}$. Si on suppose que les effets dynamiques des enchevêtrements sont faibles on trouve une valeur pour la viscosité $\eta_{\mathrm{sp}} / c \sim N c^{-1 / 2}$.

Abstract. - We discuss the conformations of linear polyions assuming that $a$ ) the corresponding uncharged chain is flexible; $b$ ) electrostatic forces dominate the monomer-monomer interactions; c) no salt is added.

1) For the dilute case (non overlapping chains) correcting a recent self-consistent calculation by Richmond [1a], we find an overall polyion size $R=N d$ which is a linear function of the polymerization index $N$ in agreement with the early work of Hermans and Overbeek, [1b], Kuhn, Kunzle, and Katchalsky $[1 c]$.

2) There is a range of very low concentration $c\left(c^{* *}<c<c^{*}\right)$ where the chains do not overlap $\left(c<c^{*}\right)$ but where the electrostatic interactions between polyions are much larger than thermal 
energies $\left(c>c^{* *}\right)$ : here we expect that the polyions build up a 3-dimensional periodic lattice; however, the detection of such an extremely dilute lattice appears difficult.

3) Practically all experiments on salt-free polyelectrolytes have been performed at concentrations $c>c^{*}$ where different chains overlap each other. To discuss this regime we restrict our attention to cases where the charge per unit length is near (or above) the condensation threshold : then a single length $\xi(c)$ characterizes the correlation; in 3 dimensions $\xi$ scales like the Debye radius associated with the counter ions. We consider several possible conformations : $a$ ) hexagonal lattice of rigid rods ; $b$ ) cubic lattice of rigid rods ; $c$ ) isotropic phase of partially flexible chains. The various rigid rod structures appear to have very similar electrostatic energies. This suggests that the isotropic phase might possibly be the most favorable. We analyse this latter phase using the same scaling methods which have recently been helpful for neutral polymer solutions (2). In the isotropic model each chain behaves like a succession of segments of size. Inside one segment electrostatic effects are important and similar to case (1) above. Between segments the interactions are screened out, and tach chain is ideal on a large scale, with radius $R(c) \sim c^{-1 / 4} N^{1 / 2}$. If we (tentatively) assume that the dynamical effects of entanglements are weak, we are than led to a viscosity $\eta_{\mathrm{sp}} / c \sim N c^{-1 / 2}$.

1. Introduction. - $1.1 \mathrm{THE}$ PROBLEM. - It has been known for a long time that linear polyelectrolytes have a large overall size and a rather stiff local conformation in dilute solution, but that they contract progressively when salt is added, or when the concentration increases. These features are summarized in the books by Tanford [3] and Oosawa [4] and in the recent articles edited by Selegny [5]. However, a detailed understanding of the conformations is still apparently lacking. In particular the statistics of overlapping polyions have been treated mainly in a rather primitive way, assuming equidistant parallel rods.

1.2 THE METHOD. - For neutral polymer solutions in good solvents the conformational problems have been clarified recently thanks to a number of neutron scattering experiments $[2,6]$ which revealed significant departures from the simple Flory-Huggins picture. In parallel with this, a rigorous theoretical analogy between polymer solutions and ferromagnets under fields has been developed by Des Cloiseaux [7] : this has allowed to transpose all our recent understanding of critical phenomena to polymers. The original Des Cloiseaux paper is cast in the esoteric language of lagrangian field theory. However, in ref. [2], we have shown how the results could be derived, and extended, by much simpler means : what is done is to start from the discussion of a single chain, following the Flory method [8], which is known to describe excluded volume effects very well. Then, the properties of semi dilute solutions (with overlapping chains) are derived from the single chain results by simple assumptions of the scaling type. The present paper applies these procedures to the problem of polyelectrolytes, where long range Coulomb forces are dominant. However, as we shall see, the sequence of arguments is much more conjectural for polyelectrolytes.

1.3 THE MODEL. - To cover these rather complex phenomena, we find it necessary to choose a very simplified model. Starting with an ideal chain of $N$ beads (unperturbed radius $R_{0}=N^{1 / 2} a$ ) we put on each bead a charge $e$. We also add a mobile counter ion of charge $-e$ in the solution. The bare Coulomb interaction between two charges $e_{1} e_{2}$ separated by $r_{12}$ is

$$
U=\frac{e_{1} e_{2}}{\varepsilon_{0} r_{12}}
$$

where $\varepsilon_{0}$ is the dielectric constant of water. Our central assumption is that all interactions other than $U$, between monomers, are negligible. If water is a good solvent for the backbone chain, these other interactions represent short range repulsions, and are indeed dominated by the longer range Coulomb forces in most practical cases. But a more dangerous possibility occurs if the polyion has an amphiphilic character - i.e. when water is a poor solvent for the backbone. Then, we could find micellar structures and even liquid crystalline phases in semi dilute solutions : these complex cases are excluded from the present model.

To make things more concrete, what we have in mind is a polyion with a repeat unit such as :<smiles>CCC(OC)S(=O)(=O)[O-]</smiles>

where the backbone oxygen ensures good water solubility even in the absence of the acid group. If we want to decrease the charge per unit length, we can use weaker acids in a partially ionized state, or longer repeat units

$$
-\left(\mathrm{C}_{2} \mathrm{H}_{4} \mathrm{O}\right)_{p-1}-\mathrm{CH}_{2} \mathrm{CHSO}_{3}^{-} \mathrm{O}-
$$

A less essential choice, required to specify the abstract model completely, amounts to treat each repeat unit either as a gaussian spring, or as one segment in a freely jointed chain : the former being closer to (ii) and the latter to (i). The distinction is not essential (except for detailed features at high charge densities). Here, for definiteness, we shall think of the backbone as a freely jointed chain. 
The charge per unit length in a fully extended conformation is then $\rho=e / a$. Instead of using $\rho$, however, we shall sometimes find it useful to introduce a characteristic length

$$
l=\frac{e^{2}}{\varepsilon_{0} k_{\mathrm{B}} T}
$$

where $T$ is the temperature and $k_{\mathrm{B}}$ is Boltzmann's constant $\left({ }^{1}\right)$. From various studies of the counterion atmosphere [4, 9-11] we know that counter ion condensation takes place when $l \geqslant a$. Thus the dimensionless coupling constant $l / a$ can vary between 0 and 1 .

The state of the solution is entirely defined in terms of the following parameters :

$a$ length of one unit,

$c$ concentration (defined here as number of units per unit volume),

$N$ polymerization index $(N \gg 1)$,

$l / a$ reduced coupling constant.

This is to be compared to the case of neutral polymer solutions [2], where the parameters are $a, c, N$, and another reduced coupling constant $v / a^{3}$ where $v$ represents the excluded volume associated with one monomer. The number of parameters is the same in both cases : thus we may hope to find a scaling theory for polyelectrolytes, although the power laws involved will obviously be very different, because of the long range nature of the forces.

2. The single chain problem. - In the present section, we assume that the different polyion chains are not overlapping and very far from each other. This will impose very low concentrations - so low that in fact most experimental techniques for conformation studies become unapplicable. However, this limit is conceptually important; it is only after understanding the single chain problem that we will be able to proceed to the more physical case of overlapping chains.

In this dilute limit each polyion occupies a certain region of the solvent with an overall size $R$ : this region we call a globule (Fig. 1). Different globules

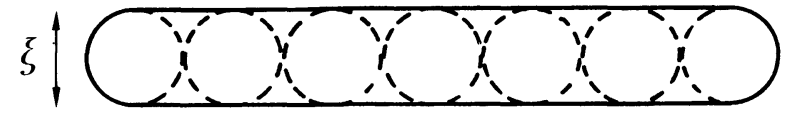

FIG. 1. - A typical polyion conformation for weak coupling in the dilute limit, as obtained from the discussion of section 2 .

are widely separated. As explained in particular in Oosawa's book [4], the counter ions are essentially uniformly spread out in the whole solution, and thus the fraction of counter ions which remains inside the globules is very small $\left({ }^{2}\right)$. Counter ion screening can

( ${ }^{1}$ For simplicity, in the following, we shall write simply $T$ instead of $k_{\mathrm{B}} T$.

$\left({ }^{2}\right)$ We shall give a direct check of this statement at the end of section 2. be omitted completely for the single chain problem (when $l / a<1$ ).

2.1 Self-CONSISTENT CalCUlation OF THE POLyiON SIZE WITH SPHERICAL SYMMETRY. - Richmond [1a] using the self-consistent field method of S. F. Edwards $[11,12]$ has found (in the absence of salt) a polyion size $R$ proportional to $N^{2 / 3}$. This is a very surprising result. The Edwards approach is a refined form of the simpler Flory approach for polymer sizes [8] which was first applied to polyelectrolytes (even before Flory) by Hermans and Oberbeek [ $1 b]$ and by Kuhn et al. [1c]. Let us first summarize briefly their argument : they assume a spherical globule of radius $R$. There are two terms in the energy of one polyion.

a) An electrostatic energy

$$
F_{\mathrm{c}} \cong \frac{(N e)^{2}}{\varepsilon_{0} R}
$$

for a spherical distribution of charge inside a radius $R\left({ }^{3}\right)$.

b) An elastic free energy

$$
F_{\mathrm{el}} \sim T \frac{R^{2}}{N a^{2}} \quad\left(N^{2} a^{2}>R^{2}>N a^{2}\right)
$$

Optimising the sum $F_{\mathrm{c}}+F_{\mathrm{el}}$ as a function of $R$ we find

$$
\begin{aligned}
R^{3} & \sim N^{3} a^{2} l \\
R & \sim N d
\end{aligned}
$$

where

$$
d=a^{2 / 3} l^{1 / 3}
$$

is an increasing function of the charge. The form (2.2) is valid for not too large elongations, and this imposes $d \ll a$ or $l / a \ll 1$. But the main result is that according to (2.3), the size $R$ is linear in $N$, and thus very different from the prediction of ref. [1a]. This is surprising since ref. [1] and eqs. (2.1-3) are based on the same ingredients.

For this reason, we have reexamined ref. [1a] in detail, and found a slight flaw in the argument, which will now be explained. We follow the lines of ref. [12]. The first unit of the chain is placed at the origin. The $n$-th unit will then be at an average distance $r(n)$ and it will feel two forces :

a) An electrostatic force $e E$ where $E$ is the electric field. Assuming that the average density of monomers around the origin $\tilde{\rho}(r)$ is spherically symmetric, we may derive the field from the Gauss theorem. Since the region inside $r(n)$ contains a charge $n e$, we have

$$
E 4 \pi r^{2}=4 \pi \frac{n e}{\varepsilon_{0}} .
$$

$\left({ }^{3}\right)$ Here we omit all numerical coefficients. 
b) An elastic force, which is on the average

$$
\frac{3 T}{a^{2}}\left[\left(\mathbf{r}_{n+1}-\mathbf{r}_{n}\right)-\left(\mathbf{r}_{n}-\mathbf{r}_{n-1}\right)\right] \cong \frac{3 T}{a^{2}} \frac{\mathrm{d} r^{2}}{\mathrm{~d} n^{2}}
$$

where we now treat $n$ as a continuous variable. These two forces must add up to zero. Using eqs. (1.2) and (2.4) this gives

$$
\frac{\mathrm{d}^{2} r}{\mathrm{~d} n^{2}}+\frac{1}{3} \frac{n d^{3}}{r^{2}}=0
$$

with the following boundary conditions

$$
\begin{array}{r}
r(n=0)=0 \\
\left.\frac{\mathrm{d} r}{\mathrm{~d} n}\right|_{N}=0
\end{array}
$$

Eq. (2.9) can readily be derived by returning to the analog of eq. (2.6) written up for the last bead, where only one elastic spring (instead of two) is involved. The behaviour near the origin is the crucial point. Richmond (probably inspired from the ThomasFermi treatment of an atom [13]) noticed that since there is one charge at the origin, the potential very near $r=0$ must be $e / \varepsilon_{0} r$. He essentially assumed that this form remained valid at small but finite $r$ thus obtaining a field $n$ times smaller than eq. (2.5) : this leads to

$$
\frac{\mathrm{d}^{2} n}{\mathrm{~d} n^{2}}+\frac{1}{3} \frac{d^{3}}{r^{2}}=0
$$

and gives $r \sim n^{2 / 3} d$. But eq. (2.7') is not justified : as soon as we have moved at a finite distance $r$, the charge inside this radius is $n e$ rather than $e$, and. eq. (2.7) holds. (This is very different from the Thomas Fermi problem, where the central nucleus brings a huge delta function peak in the charge density.)

Let us now return to the correct eq. (2.7) and turn to dimensionless variables :

$$
\left.\begin{array}{ll}
\frac{r}{N d}=u & \frac{n}{N}=x \\
\frac{\mathrm{d}^{2} u}{\mathrm{~d} x^{2}}+\frac{1}{3} & \frac{x}{u^{2}}=0 \\
u(0)=0 & \frac{\mathrm{d} u}{\mathrm{~d} x}(1)=0
\end{array}\right\} .
$$

$u(x)$ at small $x$ is nearly linear in $x$

$$
\left.\begin{array}{l}
u \rightarrow \alpha^{2} x\left[\ln \frac{1}{\alpha x}\right]^{1 / 3} \quad(x \rightarrow 0) \\
\alpha \cong 0.7
\end{array}\right\} .
$$

At the other end $(x \rightarrow 1) u$ goes to a finite value

$$
u(1) \sim 0.7 \text {. }
$$

The overall size of the polyion is thus linear in $N$

$$
R=u(1) \mathrm{d} N
$$

in agreement with the simple estimate of eq. (2.3).

2.2 Elongated Chain MODels. - The above calculations of the chain size assumed spherical symmetry, and came out with rather strong dilatations. This suggests that the chain may be in fact strongly stretched on one specific direction, and that different starting approximations should be divised. (This point has been repeatedly emphasized by Des Cloiseaux.) We shall now discuss briefly one such model, where the chain is assumed to be extended by a force $F$ acting at one extremity and a force $-F$ at the other extremity. The conformation of an ideal chain under such tensions are well known : we shall use them as our starting point in a variational calculation of the free energy, and show that the earlier estimate (2.4) of the chain length is essentially unchanged.

Our calculation assumes a weak coupling constant $(l / a \ll 1)$ such that elastic deformations are not too large. Then in the presence of the force $F$ we expect an average elongation per monomer

$$
\overline{\mathbf{a}}=\mathbf{F} \frac{a^{2}}{3 T} \text {. }
$$

The density-density correlation function for an ideal, elongated, chain has been computed by various authors [16-17] and is of the form (in terms of Fourier transforms)

$$
S(q)=2 \frac{q^{2} a^{2}}{6} \frac{1}{(\mathbf{q} \cdot \overline{\mathbf{a}})^{2}+\left(\frac{q^{2} a^{2}}{6}\right)^{2}} .
$$

We may then write the Coulomb energy as

$$
U_{\text {Coulomb }}=\frac{N}{2} \quad S(q) \frac{4 \pi e^{2}}{q^{2}} \mathrm{~d}^{3} q .
$$

And after integration over angles this becomes

$$
U_{\text {Coulomb }}=\frac{N 4 \pi e^{2}}{\varepsilon a \lambda} \int_{q} \frac{1}{q^{3}} \tan ^{-1}\left(\frac{6 \lambda}{q a}\right) q^{2} \mathrm{~d} q
$$

where

$$
\lambda=\frac{\bar{a}}{a}=-\frac{F a}{3 T} .
$$

Performing the $q$ integral we get with logarithmic accuracy

$$
U_{\text {Coulomb }}=\frac{N e^{2}}{a \lambda \varepsilon} \ln \left(\frac{\lambda R}{a}\right)
$$

where $R$ is the (unknown) chain length. We must add to (2.18) the elastic term

$$
U_{\mathrm{el}}=N \frac{3}{2} T \lambda^{2}
$$


and we must minimise the sum with respect to $\lambda$. Working only up to logarithmic accuracy we may treat the $\ln$ factor in (2.18) as a constant, and we get

$$
\lambda^{3}=\frac{l}{3 a} \ln \left(\lambda^{2} N\right)
$$

or finally a size

$$
R=N \lambda a \cong N a\left(\frac{l}{a}\right)^{1 / 3}\left(\frac{\ln (N)}{3}\right)^{1 / 3}
$$

Typically for $N \sim 10^{4}$ the factor $\left(\frac{\ln N}{3}\right)^{1 / 3}$ is of order 1.3 and thus (2.21) is not very different from the result of the spherically symmetric models.

2. 3 REMARKS ON THE SINGLE CHAIN CONFORMATION. - a) Weak coupling $l / a<1$. A qualitative picture of chains under tension has been introduced recently by one of us $\left({ }^{4}\right)$. For the case discussed here this leads to the picture of figure 1 . The chain is made of successive blobs of size

$$
\xi_{\mathrm{P}}=\frac{a^{2}}{a}=a \lambda^{-1} \sim a\left(\frac{a}{l}\right)^{1 / 3}
$$

Inside one blob the chain is nearly ideal. Between different blobs there are strong repulsions and successive blobs pile up in a straight line.

b) Strong coupling $l / a \sim 1$. - Here we are not able to make precise predictions. If we neglect all screening effects we are led to expect that the chain is fully extended. However when $l / a \sim 1$ the counterion atmosphere begins to condense around the polyion $[4,9]$ and a much more elaborate discussion is required. Thus we do not know whether the chain remains extended, or whether it becomes partly bent.

3. The lattice regime. - We begin now to include the interactions between different chains. In this section, we consider only low concentrations $c \ll c^{*}$. Again, this makes the discussion somewhat academic, since $c^{*}$ is very small for polyelectrolytes of large mass. However, since the very basis of the scaling methods is to connect the behaviour at $c \rightarrow 0$ to the behaviour at $c>c^{*}$, it is important to discuss the regime of low concentrations - at least qualitatively.

We shall argue that in a certain concentration range $c^{* *} \ll c \ll c^{*}$ the polyions should build up a periodic 3-dimensional lattice. Similar lattices have been observed with charged polystyrene spheres by Williams and Crandall $\left({ }^{5}\right)$.

$\left({ }^{4}\right)$ Pincus, P., Macromolecules, to be published

$\left({ }^{5}\right)$ Phys. Lett. 48A (1974) 225.
To discuss the lattice, we notice first that for $c<c^{*}$ the distances $D$ between neighbouring polyions will be much larger than the size $N d$ of one polyion : each polyion is point-like. Second, we remark that for $c \ll c^{*}$ the screening length $K^{-1}$ due to counter ions becomes somewhat larger than $D$. This can be seen from the equation for $K$ :

$$
K^{2}=\frac{4 \pi c e^{2}}{\varepsilon_{0} T}
$$

and from the relation between $c$ and $D$ :

$$
c=\frac{2 N}{D^{3}}
$$

(where we have chosen to put the polyion centers on a body-centered cubic lattice of cube edge $D$ ). Thus we have

$$
\begin{aligned}
K^{2} D^{2} & =\frac{8 \pi e^{2} N}{\varepsilon_{0} T D}=8 \pi \frac{N l}{D} \\
& =8 \pi \frac{l}{d}\left(\frac{l}{c^{*}}\right)^{1 / 3}=8 \pi\left(\frac{l}{a}\right)^{2 / 3}\left(\frac{c}{c^{*}}\right)^{1 / 3}
\end{aligned}
$$

where we have used the definition (2.27) for $c^{*}$. We see that for $c \ll c^{*}$ the parameter $K D$ becomes somewhat than unity : screening is weak.

Let us now focus our attention to this limit $(K D \approx 1)$. We are then dealing with point-like ions (of charge $-Q=-\mathrm{Ne}$ ) floating in a sea of uniform positive charge (density ce). At low temperatures $T$ such a system is expected to build up a body centered cubic lattice $\left({ }^{6}\right)$. At higher temperatures the lattice will melt. A qualitative understanding of the melting process can be obtained as follows : we first compute the amplitude $\delta$ of the thermal oscillations for one polyion inside the lattice, using a simple Einstein model. We then assume that the lattice is stable towards melting whenever $\delta / D$ is smaller than a fixed number $\alpha$ (typically $\alpha \sim \frac{1}{5}$ ) $\left({ }^{7}\right)$.

Let us first calculate the restoring potential $U(x y z)$ for one polyion displaced by $(x, y, z)$ form its rest position. This energy is of the form $U=-Q V$ $(x, y, z)$ where $V$ is the electrostatic potential due to all surrounding changes (polyions plus uniform sea). The cubic symmetry imposes

$$
\left.\frac{\partial V}{\partial x}\right|_{0}=\left.\frac{\partial V}{\partial y}\right|_{0}=\left.\frac{\partial V}{\partial z}\right|_{0}=0
$$

and

$$
\left.\frac{\partial^{2} V}{\partial x^{2}}\right|_{0}=\left.\frac{\partial^{2} V}{\partial y^{2}}\right|_{0}=\left.\frac{\partial^{2} V}{\partial z^{2}}\right|_{0}=\left.\frac{1}{3} \nabla^{2} V\right|_{0}=-\frac{4 \pi}{3} \frac{c e}{\varepsilon_{0}}
$$

( $\left.{ }^{6}\right)$ Wigner, E., Phys. Rev. 46 (1974) 1002; Foldy, L., Phys. Rev. B 3 (1971) 3472.

( $\left.{ }^{7}\right)$ See for example, Ziman, J., Principles of the theory of solids (Cambridge University Press, Cambridge) 1965, chap. 2. 
where we have used the Laplace equation. Finally we can write for small amplitude motions

$$
\begin{aligned}
U(x y z) & =U(0)+\frac{1}{2}\left(x^{2}+y^{2}+z^{2}\right) U^{\prime \prime} \\
U^{\prime \prime} & =\frac{4 \pi}{3} \frac{c e^{2} N}{\varepsilon_{0}} .
\end{aligned}
$$

Applying the equipartition theorem we then find a thermal average :

$$
\begin{aligned}
\delta^{2} & \equiv\left\langle x^{2}\right\rangle=\frac{T}{U^{\prime \prime}} \\
& =\frac{3}{4 \pi}(l c N)^{-1}
\end{aligned}
$$

and using eq. (3.2) we arrive at

$$
\left(\frac{\delta}{D}\right)^{2} \sim \frac{3}{8 \pi} \frac{D}{N^{2} l} .
$$

Qualitatively we may say that when $D<\frac{1}{3} N^{2} l$, the ratio $\delta / D$ is smaller than $\frac{1}{5}$ and the lattice is stable towards melting. At fixed temperature this appears as a concentration threshold $c>c^{* *}$

$$
c^{* *} \sim \frac{2 N}{\left(\frac{1}{3} N^{2} l\right)^{3}} \sim 50 N^{-5} l^{-3} .
$$

[Of course the numerical coefficient in (3.9) is very approximate.] There will indeed exist a lattice only if screening is weak, as assumed here, in the region $c \sim c^{* *}$. Thus we must have

$$
\left.K D\right|_{c=c^{* *}} \approx 1 .
$$

Returning to eq. (3.3) we see that this imposes

$$
\frac{c^{* *}}{c^{*}}<\frac{1}{(8 \pi)^{3}}\left(\frac{a}{l}\right)^{2}
$$

or, after injection of eqs. (2.27) and (3.9)

$$
N \subsetneq 8 \pi(50)^{1 / 3} \cong 150 \text {. }
$$

Eq. (3.10) explains why the lattice regime has never been found experimentally. On one hand, we need rather large values of $N$ (of order 200 or more). But, then the concentrations of interest are extremely small : for $l=a$ and $N=200$ we would have

$$
c^{* *} a^{3} \sim 10^{-5}
$$

corresponding to concentrations of order $10^{-4}$ mole/l. With concentrations which are so weak, all physical measurements become difficult. Also, to maintain the concentration of salt (or other impurities) below $c$, special precautions have to be taken.

For all these reasons, the polyelectrolyte lattice appears as a very rare possibility. It is conceptually important, however, because it brings in phase transitions which have no counterpart in neutral polymer solutions. For the latter system there is a smooth crossover between a dilute (gas like) system of separate coils, and an entangled solution. For polyelectrolytes starting from $c=0$ and increasing $c$ we may have to face two successive transitions : a cristallisation transition at $c=c^{* *}$ and a melting transition at $c \sim c^{*}$

The situation may be even more complex, because each of our polyions is not really a point, but is in fact a rod (for $l \sim a$ ). When $c$ becomes of order $c^{*}$ it may happen that the rods order along special crystallographic directions in the lattice - the problem being reminiscent of the solid phases of diatomic molecules like $\mathrm{H}_{2}$ or $\mathrm{N}_{2}$. We shall ignore these complications in the present paper.

4. Semi dilute solutions. - 4.1 CHOICE OF THE COUPLING CONSTANT. - We introduced in eq. (2.27) a concentration $c^{*}$ as the concentration of monomers inside one isolated globule. When $c>c^{*}$ different globules begin to overlap, and their conformation is strongly modified. Also counter ions must now be included in the discussion. We shall be concerned here only with the semi dilute regime, defined by the two inequalities

$$
c^{*} \ll c \ll a^{-3} .
$$

The vast majority of experiments performed on polyelectrolytes (in the absence of salts) pertain to this regime, because $c^{*}\left(\sim \frac{1}{N^{2} d^{3}}\right)$ is a very small concentration and is often unattainable in practice.

For neutral polymer solutions, as explained in the introduction, the essential parameters are $a, c, N$ and the excluded volume $v$. In good solvents, $v$ is of order $a^{3}$, and it is often convenient to focus the discussion on the case where $v=a^{3}$, as was done in ref. [2]. Qualitatively, for all finite, positive values of $v / a^{3}$ the behaviour follows the same pattern, but the condition $v=a^{3}$ avoids complicated factors of $v / a^{3}$ which make the ideas less transparent $\left({ }^{8}\right)$.

For the present polyelectrolyte problem (parameters $a, c, N$ and $l$ ) we shall make a similar simplification, assuming that $l / a=1$. Again, we expect this to maintain most features of interest. Note that this case is particularly important in practice, since it corresponds to the condensation threshold : many highly charged systems will automatically stabilize to this value of $l / a$. We also recall that in this case

$\left({ }^{8}\right)$ The complete dependence on $v$ and the behaviour near the $\Theta$ point $\left(\frac{v}{a^{3}} \rightarrow 0\right)$ has been written down in recent work by Janninck and Daoud (to be published). 
$d=a$ (as shown by eq. (2.4)) and that the critical length $r_{\mathrm{c}}$ also becomes equal to $a$ : the short range coil behaviour discussed in section 2 is thus completely eliminated.

4.2 THE heXagonal LATtice AND OTHER Related MODELS. - In the absence of salt, the electrostatic repulsions between monomers are very strong in the semi dilute regime. [In fact, as we shall see, for $l / a=1$ the repulsive energy per monomer is of order $T$ at all $c$.] The entropy contributions due to chain flexibility are smaller (since the chains remain rod like at least on certain scale $\xi(\gg a)$ which will be defined later). At first sight it is then tempting to visualise the semi dilute solutions in terms of a parallel stacking of rods : this notion has often been used in the literature $\left({ }^{9}\right)$. It is described in figure 2 .

This type of stacking would give a phase which is macroscopically uniaxial (although, of course textural defects may complicate the observation of the associated birefringence). It is not a nematic crystal, but closer to the hexagonal phases found in the soapwater system $\left({ }^{10}\right)$ : these phases have been termed canonic by F. C. Frank $\left({ }^{11}\right)$.

We have computed the energy of the hexagonal phase for infinite rods (large $N$ ) using a simple screened form for the interactions

$$
\frac{e^{2}}{r} \mathrm{e}^{-K r}
$$

where $K^{-1}$ (the Debye Huckel radius due to the counterions) is still given by eq. (3.1). The calculation (described in appendix A) gives an energy per monomer

$$
F_{\text {electrostatic }}=(-\operatorname{Ln} K a+0.084) T .
$$

We have repeated similar calculations for a cubic phase of rods (similar to the familiar $\beta$ tungsten structure of cristallographers) displayed on figure $3 b$. The interaction between chains is roughly $10 \%$ higher in the cubic phase than in the hexagonal phase (appendix A). We have then performed two other checks on the stability of the hexagonal phase.

a) We investigated the thermal fluctuations of one chain in a fixed potential due to its neighbours, allowing for both curvature energy and electrostatic energy (appendix B). The lateral displacement of

(9) Katchalsky, A., Alexandrowicz, Z., Kedem, O., in (B. Connery and R. Barradas eds) Chemical physics of ionic solutions, Wiley, New York (1966).

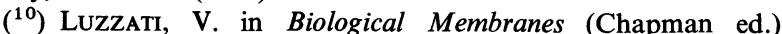
Academic Press, New York (1968); Skoulios, A., Adv. Colloid Interface Sci. 1 (1963) 79.

$\left({ }^{1}{ }^{1}\right)$ After the greek $K a v \omega v=$ rod.

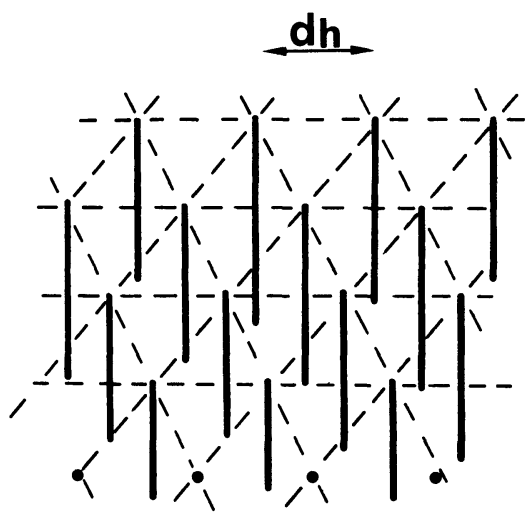

a)

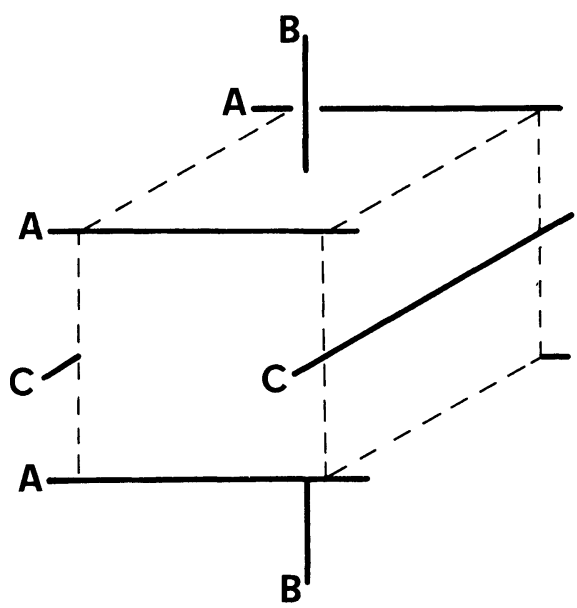

b)

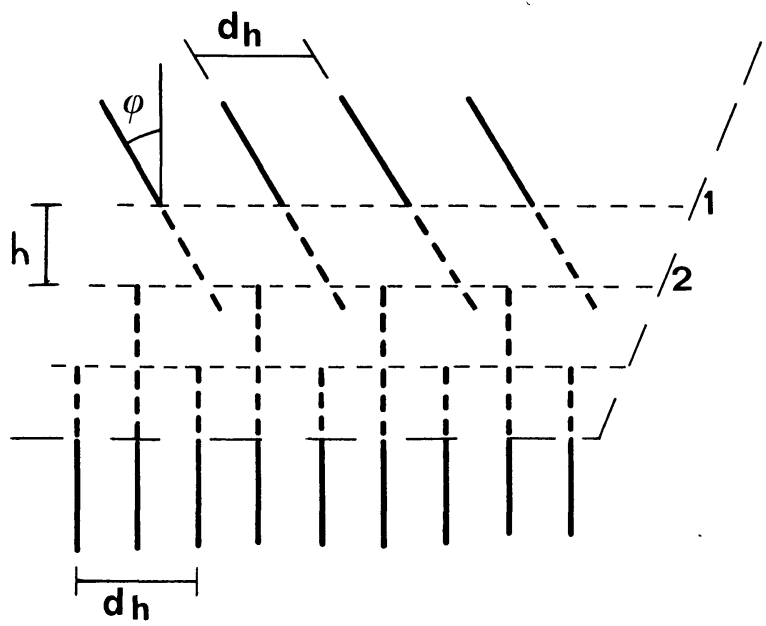

c)

Fig. 2. - a) A hexagonal lattice of rods. b) The $\beta$-tungsten structure with these families of mutually orthogonal rods $A, B, C$. c) Twist deformation of a hexagonal array of rods.

one monomer (transverse to the hexagonal axis) is small and given by

$$
\delta^{2} \sim 0.42 d_{\mathrm{h}} a
$$


where $d_{\mathrm{h}}$ is the distance between rods at rest. This implies that the relative displacement

$$
\frac{\delta^{2}}{d_{\mathrm{h}}^{2}} \sim \frac{a}{d_{\mathrm{h}}}
$$

is small (since $d_{\mathrm{h}} \gg a$ in the semi dilute regime). Thus the canonic phase appears quite stable from the point of view of individual chain fluctuations.

b) We also investigate a cooperative distortion analogous to a grain boundary (Fig. 2c). The energy calculation of this twisted interface is also given in appendix A. If our numbers are correct, the energy to create such a defect is slightly positive $\left({ }^{12}\right)$ (but independent of the twist angle).

Thus, while the hexagonal phase seems to be stable with respect to the $\beta$ tungsten structure and the deformations given in $a$ ) and $b$ ) above, we believe that it is useful to study the other possibility of random isotropic phase-consisting of overlapping partially flexible polyions. It is difficult to give a sufficiently precise calculation of the electrostatic energy of this phase to compare with the lattice structures. We conjecture however that such an isotropic situation prevails. This model may then be analysed by scaling methods which will now be described.

4.3 ThE ISOTROPIC MODEL. - a) Construction of a correlation length. - In the present picture the various polyions set up an isotropic entangled system, which is represented qualitatively on figure 3 . The local properties of this solution at fixed $c$ become independent of the chain length (of $N$ ) in the limit of large $N$ : let us first discuss this limit, i.e. restrict our attention to spatial scales $r$ smaller than the overall chain size $R(c)$. The major feature is the existence of a correlation length $\xi(c)$ which must satisfy the following requirements

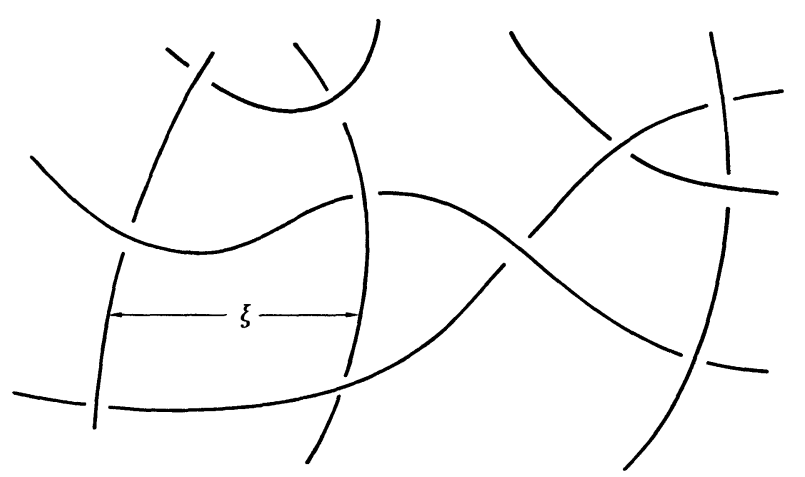

FIG. 3. - Polyion solution in the semi dilute regime. On scales $r<\xi$ the chains are nearly stiff. On scales $r \gg \Xi$ they are ideal.

(i) At fixed $c\left(>c^{*}\right), \xi(c)$ must be independent of $N$ (since it is defined in the limit $N \rightarrow \infty$ ). The only

( $\left.{ }^{12}\right)$ Approximately 6", higher parameters available being $c$ and $a, \xi$ must be of the form $\xi=a f\left(c a^{3}\right)$ where $f$ is an unknown function

(ii) For $c \rightarrow c^{*}, \xi(c)$ must become comparable to the radius $R$

$$
\xi\left(c^{*}\right) \sim R\left(c^{*}\right) \sim R(0)
$$

where $R(0)$ is the single coil size which was discussed in section 2. For the present case $(l / a=1), R(0) \sim N a$.

We now assume that $\xi / a=f$ varies like a certain unknown power of the concentration

$$
f(x)=\text { Const. } x^{-m} \text {. }
$$

Taking into account requirement (ii) this imposes

$$
\xi=R(0)\left(\frac{c^{*}}{c}\right)^{m} \quad\left(c \gg c^{*}\right) .
$$

Both $c^{*}$ and $R(0)$ depend on $N$, but $\xi$ itself must not, as explained in (i) above. Since $c^{*} \sim 1 / N^{2}$ according to eq. (2.27) and $R(0) \sim N$ we must have $m=\frac{1}{2}$

$$
\xi=R(0)\left(\frac{c^{*}}{c}\right)^{1 / 2}=\left(\frac{1}{c a}\right)^{1 / 2} .
$$

Thus $\xi(c)$ is a decreasing function of $c$, going from $R(0)$ (for $c \sim c^{*}$ ) down to the monomer size at high concentrations. Note that

(i) The length $\xi(c)$ scales like the interrod distance $d_{\mathrm{h}}$ in the hexagonal model.

(ii) The length $\xi(c)$ scales like the Debye screening radius $K^{-1}$ (eq. (3.3)) due to the counterions. This remarkable coincidence exists only in 3 dimensions.

b) The segment concept. - To reach a physical interpretation for $\xi$ we use our earlier experience with neutral polymers : at distances $r<\xi$ we expect that the single chain behaviour is maintained, while for $r>\xi$ we guess that all interactions be screened out.

Qualitatively it is very helpful to visualise the chain as a succession of segments, each carrying a number $g$ of monomers. Since, inside each segment, we have the laws of section 2 , we may write

$$
\xi \cong g d \rightarrow g a .
$$

Comparing this with eq. (3.3) for $\xi$ we see that

$$
g \cong\left(c a^{3}\right)^{-1 / 2} \cong c \xi^{3} .
$$

On the latter form we see that each volume $\xi^{3}$ is occupied by one segment on the average.

We now make the following conjecture (inspired by the neutral case) : the complete chain is an ideal necklace of $N / g$ segments successive segments being uncorrelated in their orientations. The overall radius of the chain $R(c)$ is then given by

$$
R^{2} \cong \frac{N}{g} \xi^{2} .
$$


Inserting (3.14) and (3.13) into (3.15) we find

$$
\begin{aligned}
R^{2} & \cong N a \xi \\
R(c) & \cong N^{1 / 2}\left(\frac{a}{c}\right)^{1 / 4}
\end{aligned}
$$

Eq. (4.7) may also be derived from a scaling argument, assuming that $R(c) \sim c^{-p} N^{1 / 2}$ (ideal chain behaviour on large scales) and imposing $R\left(c^{*}\right) \sim R(0) \sim N a$. This gives the equivalent form :

$$
R(c)=N a\left(\frac{c^{*}}{c}\right)^{1 / 4}
$$

We see that with our conjecture the chain radius contracts at increasing concentrations, going from $R(0) \sim N a$ to a much smaller value $\left(\sim N^{1 / 2} a\right)$ at high $c$.

c) Neutron scattering by one labeled chain. - We shall discuss two correlation functions connected with the polyions : a function $S_{1}(q)$ giving the correlations between monomers on the same chain, and a function $S(q)$ giving the correlations between any two monomers. The function $S$ is directly accessible to $\mathrm{X}$ ray or neutron studies whenever scattering by the polyion is dominant. The function $S_{1}$ is also accessible through the use of a few deuterated polyion chains, immersed in a solution of normal (protonated) polyion chains.

Let us start for instance from the one chain function $S_{1}(q)$. When the wave vector $q$ is larger than $1 / \xi$ we probe the inside of a segment, and we expect to find the same scattering law which would obtain in a single chain. Since $l \sim d \sim a$ this is

$$
S_{1}(q)=\frac{\pi}{q a} \quad(q \xi>1) .
$$

On the other hand, at small $q \xi$, our conjecture predicts a form of $S_{1}$ based on an ideal chain, made of $N / g$ segments of size $\xi$. The Debye law for this case is of the form

$$
S_{1}(q) \cong \frac{g}{q^{2} \xi^{2}} \quad\left(\frac{1}{\xi}>q>\frac{1}{R}\right)
$$

(apart from a numerical coefficient which the scaling laws do not predict). Note that for $q \sim 1 / \xi$ both equations $(3.8,9)$ give qualitatively the same result

$$
S_{1}\left(\xi^{-1}\right) \sim \frac{\xi}{a} \sim g .
$$

d) Coherent scattering. - Let us now turn to the complete monomer-monomer correlation function $S(q)$. At high $q$, we are dealing with correlations inside one segment. Since, as noticed earlier, different segments do not overlap on the average we must have $S=S_{1}$, in this regime

$$
S(q)=\frac{\pi}{q a} \quad(q \xi \geqslant 1) .
$$

At low $q$, we do not know the form of $S(q)$, but we may relate $S(0)$ to the osmotic compressibility

$$
S(0)=T \frac{\mathrm{d} c}{\mathrm{~d} \pi_{\mathrm{p}}}
$$

where $\pi_{\mathrm{p}}$ is the osmotic pressure contribution due to the polyions $\left({ }^{13}\right)$. As already mentioned, the electrostatic energy per monomer is of order $T$. The entropic terms due to chain distortions are $g$ times smaller (since each segment acts as one unit) and do not contribute very much to the free energy. We may expect that the pressure $\pi_{\mathrm{p}}$ scales like the free energy per unit volume and is thus of order

$$
\pi_{\mathrm{p}}=\text { Const. } c T \text {. }
$$

(Note incidentally that this is dimensionally comparable to the counterion pressure.) Thus we are led to assume that $S(0)$ is of order unity, i.e. very small in comparison with $S(q \xi) \sim g$. This suggests that the plot of $S(q)$ versus $q$ should show a maximum at $q \sim$ Const. $\xi^{-1}$. Such a peak may be interpreted as demonstrating the short range order arising from the hard-sphere like repulsion between segments. The few neutron data presently available give us contradictory evidence on this point. In one case $\left({ }^{14}\right)$ (with carboxymethyl cellulose) no maximum has been found. In another case $\left({ }^{15}\right)$ (with metacrylic acids of low mass) a peak is apparently observed - but it might be due to completely different effects (segregation of the hydrophobic portions). More detailed experiments are clearly needed.

It is not possible to write down a scaling form for $S(q)$ if we want to satisfy both $S(0) \cong 1$ and eq. (4.11) at large $q$. However, a scaling form may be restored in the limit $g \gg 1-$ i.e. in a limit where $S(0)$ is negligible when compared to $S\left(\xi^{-1}\right)$. Then we can postulate

$$
S(q)=g f(q \xi)
$$

where $f(x)$ is a dimensionless, universal function of $x$ (i.e., is the same at all concentrations in the semi dilute regime). For large $x, f \sim 1 / x$. For small $x, f$ goes to zero. The function $f(x)$ would have a maximum for $x \sim 1$.

5. Concluding remarks. -5.1 Remarks ON THE ONE CHAIN PROBLEM. - It is probably wise to emphasize first the limitations of the present work : our analysis of the conformations of a single polyion is based on approximations of the Flory type [5] which give good results for the excluded volume problem (i.e. for short range repulsions). But the success of the Flory method is due to a delicate

$\left({ }^{13}\right)$ To define $\pi_{p}$ it is convenient to introduce a model where the coulomb interactions between polyion charges are screened with a radius $K^{-1}$, and where the counterions are eliminated from the model.

(14) Moan M. and Wolff, C., Polymer 16 (1975) 776.

( ${ }^{15}$ ) Cotton, J. P. and MoAn, M., J. Physique Lett. to be publish- 
compensation of overestimates in the two terms of the free energy (elastic + repulsive) [18] : it is by no means certain that this compensation is maintained when we are dealing with long range forces. Thus our discussion of section 2 should be supplemented by a more rigorous approach.

It is interesting in this connection to see if there is a critical dimensionality $d_{\mathrm{c}}$ above which a charged chain would remain essentially unstretched.

One way to ascertain this is based on perturbation expansions for the end to end radius $R$ as a function of the charge $e$, for small charge : this is formally similar to the Fixman calculation for neutral chains $\left({ }^{16}\right)$. For an arbitrary dimensionality $d\left({ }^{17}\right)$ the Coulomb interaction between two charges $e$ separated by a distance $r$ is proportional to

$$
\frac{e^{2}}{\varepsilon r^{d-2}}
$$

The expansion of $R\left(e^{2}\right)$ is then of the form

$$
R^{2}=R_{0}^{2}\left[1+\text { Const. } \frac{e^{2} N^{2}}{\varepsilon R_{0}^{d-2} T}+\cdots\right] .
$$

Since $R_{0}=a N^{1 / 2}$ the expansion parameter is small when $d>6$. Above $d=6$ the deviations from ideality are minor. Below $d=6$ they are essential; the radius $R$ must be computed by more sophisticated methods $\left({ }^{18}\right)$. It is expected to be of the form $R=a N^{v(d)}$ with $v>\frac{1}{2}$.

5.2 QUESTIONS CONCERNING THE SEMI DILUTE REGIME. - We have been led to consider the isotropic model as the most plausible candidate for the representation of semi dilute solutions. However we are faced with many pitfalls.

a) The decision between hexagonal and isotropic models is delicate, and might require inclusion of a more sophisticated discussion of counterion atmospheres $[4,5]$.

b) Another possibility which is not ruled out is to have a nematic type of order - without any hexagonal lattice, but with one preferred orientation for the rods. Nematic phases have been found long ago for certain rod like molecules like helical polypeptides [19]. It may be that the flow birefringence data (showing an extinction angle progressively reduced by larger shear rates) represent in fact the progressive elimination of defects and alignment of a nematic texture : clearly many more experiments in this field are required.

c) Even if the isotropic model is right, we may still question our further conjecture on the shape of the chains : is the radius of gyration proportional

(16) See Zimm, B. H., Stockmayer, W. H. and Fixman, M., J. Chem. Phys. 21 (1953) 1716.

$\left({ }^{17}\right)$ In this section, $d$ is the dimensionality, and should not be confused with the length $d$ of section 2 .

$\left({ }^{18}\right)$ The corresponding renormalisation group is currently being analysed by us, together with P. Pfeuty. to $N^{1 / 2} c^{-1 / 4}$ (as proposed here) or are the rods more strongly extended ? A neutron experiment with labeled chains would solve the problem. At present the only available (and rather preliminary) data are for unlabeled chains of carboxymethyl celluloses $\left({ }^{19}\right)$. They do show a crossover length $\xi$ which decreases with concentration; but the detailed power laws are still to be checked. Also the plots of $S(q)$ do not show the maximum at $q \xi \sim 1$ which we would expect.

d) The viscosity $\eta$ of polyelectrolyte solutions remains a mystery.

- The main results (in zero salt) concern extrapolations at $c \rightarrow 0$ which seem to confirm that the single chain radius $R$ is linear in $N$ as expected.

- In the semi dilute regime, the usual finding is $[3,4,5]$

$$
\frac{\eta-\eta_{0}}{\eta_{0} c} \sim f(N) c^{-1 / 2}
$$

where $f(N)$ is essentially unknown, although some early data by Eisenberg suggest $f \sim N$. A dynamical scaling analysis $\left({ }^{20}\right)$ based on the reptation model would give

$$
\frac{\eta-\eta_{0}}{\eta_{0} c} \sim N^{3} c^{1 / 2}
$$

in strong disagreement with (5.3).

It is in fact, striking that $\eta(c, N)$ increases much more slowly with $c$ and $N$ than for neutral solutions. Two lines of thought can be proposed here

(i) Either the polyelectrolyte chains are not entangled - even when they overlap strongly $\left(c>c^{*}\right)$. They might, for instance, be locally parallel to each other even if they have the coil radius $R$ of eq. (4.6).

(ii) Or the entanglement contribution to the energy of a distorted network is small compared to the electrostatic contribution. This certainly has some truth, but the ratio of these two energies is of order $1 / \mathrm{g}$ and probably not small enough to explain the data.

Along the lines of (i) we can make the following remark : if we assume that entanglement effects are negligible, we may try to predict the viscosity by a simple free draining argument of the Rouse type [20]. This gives

$$
\eta-\eta_{0}=\frac{1}{36} c f_{1} R_{\mathrm{G}}^{2}
$$

where $f_{1}$ is the friction factor for one monomer against the solvent. Inserting our form (4.6) for $R_{\mathrm{G}}$ and a monomer hydrodynamic radius $a_{\mathrm{H}}$ (such that $f_{1}=6 \pi \eta_{0} a_{\mathrm{H}}$ ) we would get

$$
\frac{\eta-\eta_{0}}{\eta_{0} c}=\frac{\pi}{6} a_{\mathrm{H}} a \xi N \sim N c^{-1 / 2}
$$

The concentration dependence would agree with eq. (5.3). The dependence on $N$ remains to be checked.

$\left({ }^{19}\right)$ Moan, M. and Wolff, C., Polymer 16 (1975) 776.

$\left({ }^{20}\right)$ F. BROCHARD, unpublished. 
Acknowledgments. - We have greatly benefited from conversations with G. Jannink, L. Ter Minassian-Saraga, M. Moan, M. Rinaudo and C. Wolff.

\section{APPENDIX A}

The electrostatic lattice energies. - Let us first calculate the electrostatic energy between two infinite parallel chains separated by a distance $\rho$, using the screened interaction (4.1).

We take the $z$-axis parallel to the chains; thus the energy per monomer is given by

$$
\frac{F^{\prime}(\rho)}{T}=\frac{e^{2}}{T \varepsilon_{0} a} \int_{-\infty}^{\infty} \frac{\mathrm{e}^{-K \sqrt{\rho^{2}+z^{2}}}}{\sqrt{\rho^{2}+z^{2}}} \mathrm{~d} z=\frac{2 l}{a} K_{0}(K \rho)
$$

where $K_{0}(K \rho)$ is the modified Bessel function, which in all our calculation can be approximated by its asymptotic expansion for $K \rho>1$. (We also take $l=a$; i.e. strong coupling limit.) Then

$$
\frac{F_{\text {electrostatic }}^{\prime}(\rho)}{T} \cong \sqrt{\frac{2 \pi}{K \rho}} \mathrm{e}^{-K \rho} \text {. }
$$

The hexagonal lattice energy, figure $2 a$ is calculated from (A.2) by adding the energy contribution of the chains around a given one

$$
\begin{aligned}
\frac{F_{\text {electrostatic }}}{T}=\frac{z_{1}}{2 T} & F_{\text {electrostatic }}^{\prime}\left(\rho_{1}\right)+ \\
& +\frac{z_{2}}{2 T} F_{\text {electrostatic }}^{\prime}\left(\rho_{2}\right)+\cdots
\end{aligned}
$$

where $\frac{1}{2} F^{\prime}(\rho)$ gives us the contribution of a chain at a distance $\rho, z_{1}=6, z_{2}, \ldots$ are the number of nearest, next-nearest neighbours and $\rho_{1}=d_{\mathrm{h}}, \rho_{2}=\sqrt{3} d_{\mathrm{h}}, \ldots$ the corresponding distances. The nearest neighbour distance $d_{\mathrm{h}}$ is determined by the condition

$$
a A C=1
$$

where $c$ is the concentration and $A$ the area per chain in semi dilute regime; in the hexagonal phase

$$
A_{\mathrm{h}}=\frac{\sqrt{3}}{2} d_{\mathrm{h}}^{2}
$$

and

$$
K d_{\mathrm{h}}=\sqrt{\frac{8 \pi}{\sqrt{3}}}=3.809 .
$$

The most important contribution to the interaction energy is the nearest neighbour energy, then we will only take the first term in eq. (A.3). Substitution of $d_{\mathrm{h}}$ in eqs. (A.2) and (A.3) gives us the interaction energy

$$
\frac{F_{\mathrm{h}}}{T}=0.084 \text {. }
$$

To obtain the total energy we must add the self-energy of a chain

$$
\frac{F_{\text {self }}}{T}=-\frac{l}{a} \operatorname{Ln}(K a) .
$$

Then in the strong coupling limit $(l=a)$ we obtain the total energy per monomer of a chain in hexagonal phase eq. (4.2).

In order to calculate the energies in $\beta$-tungsten and the grain boundary structures, figures $2 b, 2 c$ we have computed the interaction energy between two chains separated a distance $\delta$ and making an angle $\varphi$, e.g. an arbitrary chain on plane 1 , and a chain in plane 2 , figure $2 c$. The interaction energy is calculated in the following manner : we consider a monomer $i$ on chain 1 and a monomer $\mathrm{j}$ on chain 2 separated a distance $r$, the screened Coulomb interaction is

$$
u(r)=\frac{e^{2}}{\varepsilon_{0} r} \mathrm{e}^{-K r} .
$$

The energy per unit length $E_{12}$ between the pair of chains is then

$$
E_{12}(\delta)=\int_{\delta}^{\infty} u(r) p(r) \mathrm{d} r
$$

where $p(r)$ is the probability per unit length of finding monomers (i, j) separated by $r$.

To calculate $p(r)$ let us take the $z$-axis along chain 1 , $S_{1}$ is the distance between the origin and monomer i; the coordinates or monomer $\mathrm{j}$ are $\left(S_{2} \sin \varphi, \delta, S_{2} \cos \varphi\right)$. Then the distance $r$ between the pair $(i, j)$ can be written as

$$
r^{2}=\delta^{2}+S_{1}^{2}+S_{2}^{2}-2 S_{1} S_{2} \cos \varphi .
$$

Eq. (A.9) represents an ellipse in the plane $S_{1} S_{2}$ with an area

$$
\Sigma(r)=\frac{\pi\left(r^{2}-\delta^{2}\right)}{|\sin \varphi|}
$$

The probability $p(r)$ is then

$$
p(r)=\frac{1}{a^{2}} \frac{\mathrm{d} \Sigma(r)}{\mathrm{d} r} .
$$

Finally

$$
\frac{F_{12}}{T}=\frac{2 \pi}{a K|\sin \varphi|} \mathrm{e}^{-K \delta} ; \quad(l=a) .
$$

In figure $2 c$ we note that there exist many crossings of this type between the chains on plane 1 and the chains in plane 2 , in fact there are $|\sin \varphi| \mid \rho$ per unit length where $\rho$ is the distance between chains on a 
plane (e.g. planes 1 or 2). Then the interaction energy per monomer is

$$
\frac{F^{\prime}}{T}(\delta, \rho)=E_{12}(\delta) \frac{|\sin \varphi|}{\rho} a=\frac{2 \pi}{K \rho} \mathrm{e}^{-K \delta} .
$$

We note that the interaction energy is independent of $\varphi$.

Now consider an arbitrary chain in the $\beta$-tungsten structure, figure $2 b$. The nearest neighbours make an angle $\varphi=\pi / 2$, so their energy contribution is proportional to eq. (A.13) :

$$
\frac{z_{1}}{2} \frac{F^{\prime}\left(\delta_{1} \rho_{1}\right)}{T}
$$

where $z_{1}=2$ is the number of nearest neighbours and $F^{\prime}\left(\delta_{1} \rho_{1}\right) / 2$ gives us the contribution of a chain, and we note that the distances between planes and chains are emual : $\delta_{1}=\rho_{1}=d_{\beta}$. The next nearest neighbours are parallel and the energy is proportional to (A.2). Then the lattice interaction energy can be written as

$$
\frac{F_{\beta}}{T}=\frac{z_{1}}{2 T} F^{\prime}\left(d_{\beta}, d_{\beta}\right)+\frac{z_{2}}{2 T} F_{\text {electrostatic }}^{\prime}\left(\rho_{2}\right)+\cdots .
$$

The nearest neighbour distance is calculated from eq. (A.4)

$$
A_{\beta}=\frac{4}{3} d_{\beta}^{2} \quad \text { and } \quad K d_{\beta}=\sqrt{3 \pi}=3.069 .
$$

To compare hexagonal and $\beta$-tungsten lattice energies we take only the nearest neighbours contribution in eq. (A.14). (The self energy of the chains is of course the same in the two phases.)

$\frac{F_{\beta}}{F_{\mathrm{h}}}=\frac{\sqrt{2 \pi}}{3} \frac{1}{K d_{\beta}} \frac{d_{\mathrm{h}}}{d_{\beta}} \exp \left[-K d_{\beta}\left(1-\frac{d_{\mathrm{h}}}{d_{\beta}}\right)\right]=1.11$.

We see that the $\beta$-tungsten energy is about $10 \%$ greater than the hexagonal phase energy.

Let us now consider the grain boundary structure which is obtained from the hexagonal structure by the cooperative distortion of twisting a plane by an arbitrary angle $\varphi$ as shown in figure $2 c$. The interaction energy of a chain on plane 1 with the chains in plane 2 will be proportional to eq. (A.13), in fact

$$
F_{\varphi}(\delta, \rho)=\frac{1}{2} F^{\prime}(\delta, \rho) \text {. }
$$

The distance between the planes 1 and 2 is

$$
\delta=h\left(h=\frac{\sqrt{3}}{2} d_{\mathrm{h}}\right)
$$

and the distance between the chains in each plane is $\rho=d_{\mathrm{h}}$ as indicated in figure $2 c$. Then

$$
\frac{F_{\varphi}}{T}=\frac{\pi}{K d_{\mathrm{h}}} \mathrm{e}^{-K h}
$$

Now we compare the energy $F_{\varphi}$ with the interaction energy, the chain on plane 1 would have in the undistorted phase. In the undistorted phase the considered chain in plane 1 is parallel to the chains in plane 2 and it has $z^{\prime}=2$ nearest neighbours at a distance $d_{\mathrm{h}}$, then the interaction energy is given by eq. (A.2) which we call

$$
\begin{aligned}
& F_{\|}=\frac{z^{\prime}}{2} F_{\text {electrostatic }}^{\prime}\left(d_{\mathrm{h}}\right) \\
& \frac{F_{\|}}{T}=\sqrt{\frac{2 \pi}{K d_{\mathrm{h}}}} \mathrm{e}^{-K d_{\mathrm{h}}} .
\end{aligned}
$$

A comparison with eq. (A.17) gives us

$$
\frac{F_{\varphi}}{F_{\|}}=\sqrt{\frac{\pi}{2 K d_{\mathrm{h}}}} \exp \left[K d_{\mathrm{h}}\left(1-\frac{\sqrt{3}}{2}\right)\right]=1.06 .
$$

We see the interaction energy in $\varphi$ structure is greater than the undistorted phase energy, but all the interaction energies are of the same order of magnitude.

\section{APPENDIX B}

Thermal fluctuations in the hexagonal phase.-We describe the calculation of the lateral chain displacement in the hexagonal phase produced by thermal fluctuations.

The total energy per monomer of a deformed chain in the hexagonal phase has two main terms : an electrostatic repulsion produced by the chains in the immediate neighbourhood, which opposes to lateral deformation, and the curvature energy. The first term can be calculated from

$$
F_{\text {rep }}=\frac{1}{2}\left(\frac{\partial^{2} V}{\partial x^{2}}(\delta x)^{2}+\frac{\partial^{2} V}{\partial y^{2}} \delta y^{2}\right)
$$

where $\delta x$ and $\delta y$ are the lateral displacements and $V$ is the potential produced by the neighbouring chains

$$
\begin{aligned}
& \nabla^{2} V=K^{2} V_{\text {rep }} \\
& V_{\text {rep }}=z_{1} F_{\text {electrostatic }}\left(d_{\mathrm{h}}\right)
\end{aligned}
$$

$z_{1}$ is the number of neighbours $\left(z_{1}=6\right)$ and $F_{\text {electrostatic }}$ is given by (A.2), then eq. (B. 1) reduces to

$$
\frac{F_{\text {rep }}}{T}=\frac{x^{2}}{4} \frac{V_{\text {rep }}}{T} \delta_{n}^{2} .
$$


The curvature energy per monomer $(l=a)$ for an isolated chain is given by

$$
\frac{F_{\mathrm{c}}}{T}=\frac{1}{8 K^{2}}\left(\frac{\partial^{2} \delta x_{n}}{\partial Z^{2}}\right)^{2}+\left(\frac{\partial^{2} \delta y_{n}}{\partial Z^{2}}\right)^{2} .
$$

Taking Fourier components for the displacements

$$
\delta x_{n}(q)=\sum_{n} \mathrm{e}^{i \mathbf{q} \cdot r n} \delta x_{n}
$$

and adding (D.4) (D.5) we obtain the total energy

$$
\frac{F_{\text {tot }}}{T}=\frac{1}{2 N} \sum_{n} \sum_{q}\left[\left(\frac{V_{\text {rep }}}{T} K^{2}+\frac{1}{2} \frac{q^{4}}{K^{2}}\right) \delta x_{n}^{2}(q)\right] \text {. }
$$

The thermal average gives us the value of $\left\langle\delta x_{n}^{2}(q)\right\rangle$

$$
\left\langle\delta x_{n}^{2}(q)\right\rangle=\frac{N}{\frac{V_{\text {rep }}}{T} K^{2}+\frac{1}{2} \frac{q^{4}}{K^{2}}}
$$

and

$$
\left\langle\delta x_{n}^{2}\right\rangle=\frac{1}{N} \sum_{q}\left\langle\delta x_{n}^{2}(q)\right\rangle
$$

then

$$
\left\langle\delta^{2}\right\rangle=\frac{a K^{-1}}{2(2)^{1 / 4}}\left(\frac{V_{\mathrm{rep}}}{T}\right)^{-3 / 4}=0.420 a d_{\mathrm{h}} .
$$

\section{References}

[1a] Richmond, P., J. Phys. A 6 (1973) L 109.

[1b] Hermans, J., Overbeex, J., Recents Travaux Chimiques 67 (1968) 761.

[1c] Kuhn, W., Kunzle, D., Katchalsky, A., Helv. Chim. Acta 31 (1948) 1994.

[2] Daoud, Cotton, Farnoux, Jannink, Sarma, Benoit, DuplesSIX, Picot, DE Gennes, Macromolecules 8 (1975) 804.

[3] TANFord, C., Physical Chemistry of Macromolecules Wiley (NY) 1961.

[4] Oosawa, F., Polyelectrolytes, M. Dekker (NY) 1971.

[5] Selegny, E., Mandel, M., Strauss, U., Polyelectrolytes D. Reidel. Dordrecht 1974.

[6] Farnoux, B., Thèse, Strasbourg 1975 (available from S.P.S.R.M., C.E.N.-Saclay, Orme des Merisiers, B.P. no 2, 91 Gif-sur-Yvette, France).

[7] des Cloiseaux, J., J. Physique 36 (1975) 281.

[8] Flory, P., Principles of polymer chemistry (Cornell Un. Press) Ithaca (N.Y.) 1967.

[9] Manning, G. S., J. Chem. Phys. 5 (1969) 924, 934, 3249.
[10] a) JaCkson, J., Corriell, S., J. Chem. Phys. 40 (1964) 1460 b) Mc Gillivray, A. D., Winkelman, J., J. Chem. Phys. 45 (1966) 2184.

[11] Edwards, S. F., Proc. Phys. Soc. 85 (1965) 813.

[12] De Gennes, P. G., Rep. Prog. Phys. 32 (1969) 187.

[13] See for instance ScHIFF, L. I., Quantum mechanics (Mc Graw Hill) 1951.

[14] Debye, P., J. Phys. Colloid. Chem. 51 (1947) 18.

[15] Kratry, O., POROD, G., Récents travaux chimiques 68 (1949) 1106.

[16] de Gennes, P. G., Physics 3 (1967) 37.

[17] BENOIT, H., private communication.

[18] See for instance DE GENNES, P. G. (to be published in Macromolecules);

Des Cloiseaux, J. (to be published in J. Physique).

[19] Robinson, C., Discuss. Faraday Soc. 25 (1968) 29.

[20] Rouse, P. E., J. Chem. Phys. 21 (1953) 1272.

[21] Moan, M., Wolff, C., Ober, R., Polymer 16 (1975) 781 ; Moan, M., Wolff, C., Polymer 16 (1975) 776. 